\title{
Perirhinal cortex resolves feature ambiguity in configural object recognition and perceptual oddity tasks
}

\author{
Susan J. Bartko, ${ }^{1,4}$ Boyer D. Winters, ${ }^{1}$ Rosemary A. Cowell, ${ }^{2}$ Lisa M. Saksida, ${ }^{1,3}$ \\ and Timothy J. Bussey ${ }^{1,3}$ \\ ${ }^{7}$ Department of Experimental Psychology, University of Cambridge, Cambridge CB2 3EB, United Kingdom; ${ }^{2}$ LEAD-CNRS, \\ Université de Bourgogne, Pôle AAFE, Esplanade Erasme 21065 Dijon, France; ${ }^{3}$ MRC and Wellcome Trust Behavioural and Clinical \\ Neuroscience Institute, University of Cambridge, Cambridge CB23EB, United Kingdom
}

\begin{abstract}
The perirhinal cortex (PRh) has a well-established role in object recognition memory. More recent studies suggest that PRh is also important for two-choice visual discrimination tasks. Specifically, it has been suggested that PRh contains conjunctive representations that help resolve feature ambiguity, which occurs when a task cannot easily be solved on the basis of features alone. However, no study has examined whether the ability of PRh to resolve configural feature ambiguity is related to its role in object recognition. Therefore, we examined whether bilateral excitotoxic lesions of PRh or PPRh (perirhinal plus post-rhinal cortices) in the rat would cause deficits in a configural spontaneous object recognition task, and a configural simultaneous oddity discrimination task, in which the task could not be solved on the basis of features, but could only be solved using conjunctive representations. As predicted by simulations using a computational model, rats with PPRh lesions were impaired during a minimal-delay configural object recognition task. These same rats were impaired during a zero-delay configural object recognition task. Furthermore, rats with localized PRh lesions were impaired in a configural simultaneous oddity discrimination task. These findings support the idea that PRh contains conjunctive representations for the resolution of feature ambiguity and that these representations underlie a dual role for PRh in memory and perception.
\end{abstract}

Although the perirhinal cortex (PRh) has a well-established role in object recognition memory (Meunier et al. 1993; Suzuki et al. 1993; Mumby and Pinel 1994; Aggleton et al. 1997; Winters et al. 2004; Winters and Bussey 2005), it has more recently been suggested that PRh is also important for two-choice visual discrimination tasks. Specifically, it has been suggested that PRh contains conjunctive representations that help resolve feature ambiguity, which occurs when a task cannot easily be solved on the basis of features alone (Murray et al. 2007). The biconditional discrimination $(\mathrm{AB}+, \mathrm{CD}+, \mathrm{BC}-, \mathrm{AD}-)$ is a commonly used configural task that contains a high level of feature ambiguity: Since each of the features A, B, C, and D are rewarded as often as not, these features alone cannot be used to solve the task. However, the conjunctions $\mathrm{AB}$ and $\mathrm{CD}$ are always rewarded and the conjunctions $\mathrm{BC}$ and $\mathrm{AD}$ are never rewarded; therefore, these conjunctions can be used to solve the task. Damage to PRh has been shown to disrupt performance in the biconditional discrimination and other configural tasks (Buckley and Gaffan 1998; Eacott et al. 2001; Bussey et al. 2002; Saksida et al. 2007). However, these functions mediated by $\mathrm{PRh}$-object recognition and the resolution of feature ambiguity in configural discrimination tasks-are usually regarded separately. Indeed, it has been suggested that apparently different functions of PRh may be carried out through distinct mechanisms mediated by distinct neural populations within PRh (Bogacz et al. 2001). However, a recent computational model shows how the existence of conjunctive (configural) representations in PRh can explain delay-dependent and list length-dependent impairments in object recognition memory

\footnotetext{
${ }^{4}$ Corresponding author.
}

E-mail sjb237@cam.ac.uk; fax 44-1223-333564.

Article is online at http://www.learnmem.org/cgi/doi/10.1101/lm.749207. following lesions of PRh (Cowell et al. 2006). Furthermore, a recent behavioral study has shown how perceptual and mnemonic functions of PRh might interact, revealing impairments in object recognition following PRh lesions when objects were manipulated to increase their perceptual similarity (Bartko et al. 2007; see also Eacott et al. 1994). However, no study has used a similar analysis to determine whether the ability of PRh to resolve feature ambiguity in configural discriminations is related to its role in object recognition.

Therefore in the present study, we modified the object recognition and perceptual oddity judgment tasks used by Bartko et al. (2007) to produce configural versions of these tasks. We based the configural object recognition and configural oddity tasks on the maximum feature ambiguity discrimination task (the biconditional task) used in Bussey et al. (2002), on which monkeys with PRh lesions were severely impaired. To convert the biconditional task $(\mathrm{AB}+, \mathrm{CD}+, \mathrm{BC}-, \mathrm{AD}-)$ to an object recognition task, the correct compound object $\mathrm{AB}$ was used as the novel stimulus in the object recognition task, and the incorrect compounds $\mathrm{BC}$ and $\mathrm{AD}$ were used as the familiar objects (i.e., as samples). Thus, $\mathrm{BC}$ and $\mathrm{AD}$ were presented in the sample phases (and thus would be familiar when presented in the choice phase), and $\mathrm{AB}$ was presented as the novel stimulus in the choice phase. As a result, all of the features $(\mathrm{A}, \mathrm{B}, \mathrm{C}$, and $\mathrm{D})$ presented in the choice phase were familiar, and so the novel stimulus could not be determined using the features alone; only the conjunction of features was novel. To construct a control task, the minimum feature ambiguity condition $(\mathrm{AB}+, \mathrm{CD}+, \mathrm{EF}-, \mathrm{GH}-)$ used in Bussey et al. (2002), in which the discrimination contained no ambiguous features and could be solved on the basis of individual features alone, was converted to an object recognition task. Since the compound $\mathrm{AB}$ was rewarded in the minimum feature ambi- 
guity condition of the discrimination task, and EF and GH were unrewarded, we presented $\mathrm{AB}$ as the novel stimulus and $\mathrm{EF}$ and $\mathrm{GH}$ as the familiar stimuli in the control object recognition task. Therefore, in the control condition of the object recognition task, the novel stimulus $(\mathrm{AB})$ contained features that were not presented in the sample stimuli (EF and $\mathrm{GH}$ ). In this condition, a judgment of novelty could now be made on the basis of features alone. In the configural oddity task, five object pairs were presented simultaneously, an odd object $(\mathrm{AB})$, and two different pairs of identical objects (CB 1, CB 2, AD 1, and AD 2). Again, the odd object could not be identified on the basis of individual features; only the conjunction of features distinguishes the odd object from the other, "repeating" objects.

In experiment 1 , simulations using the computational model of Cowell et al. (2006) are provided to make explicit our predictions for the configural object recognition experiment (experiment 2) and the rationale behind these predictions. In experiment 2, we examined the behavior of rats with PPRh (perirhinal plus post-rhinal cortex) lesions using a minimal delay between the two sample and choice phases of the spontaneous configural object recognition task using Lego stimuli. In experiment 3, we examined the behavior of rats with PPRh lesions in a zero-delay configural object recognition paradigm using junk object stimuli. The modified paradigm allowed us to test under conditions of zero delay; the zero-delay condition in object recognition experiments is usually considered to have little or no memory load and has been used as an assay of perceptual function (Eacott et al. 1994; Buffalo et al. 1999, 2000; Holdstock et al. 2000; Levy et al. 2005). In experiment 4, we examined the effects of selective PRh lesions in the configural simultaneous oddity discrimination task. During the configural oddity task, stimuli were presented to the rat simultaneously, so that stimuli were always present and there was no requirement to remember the stimuli across a delay.

\section{Results}

\section{Experiment 1: Model simulation}

In experiment 1 , we present a simulation that demonstrates the predictions of the model (for the architecture of the model, see Fig. 1A) (see also Cowell et al. 2006) for the effects of damage to PRh on recognition memory for novel conjunctions of features. As shown in Figure 1B, networks in groups Intact and Lesion performed well on object recognition in the control condition and both groups performed poorly in the configural condition. However, a clear group difference, not seen in the control condition, was revealed between the groups in the configural condition: Group Intact was still able to discriminate the novel and familiar stimuli, whereas networks in group Lesion were unable to perform the discrimination. Thus, the model predicts that recognition of a novel conjunction of features will be impaired in subjects with PRh lesions relative to the performance of control subjects. This prediction of the model arises because, whereas the intact networks can represent the conjunction of stimulus features corresponding to the whole object as well as simple conjunctions corresponding to individual features (see Fig. 1C), the lesioned networks can represent only individual features. In the control condition, two objects are presented to networks in the choice phase-one of which is composed entirely of familiar features and one of which contains only novel features, and both layers of the model are able to discriminate the stimuli on the basis of familiarity. On the caudal layer, where low-dimensional conjunctions of stimuli are represented separately, both features are sharply tuned for the familiar stimulus whereas both are coarsely tuned for the novel stimulus. Similarly, on the PRh layer, the single conjunctive representation of the familiar stimulus is
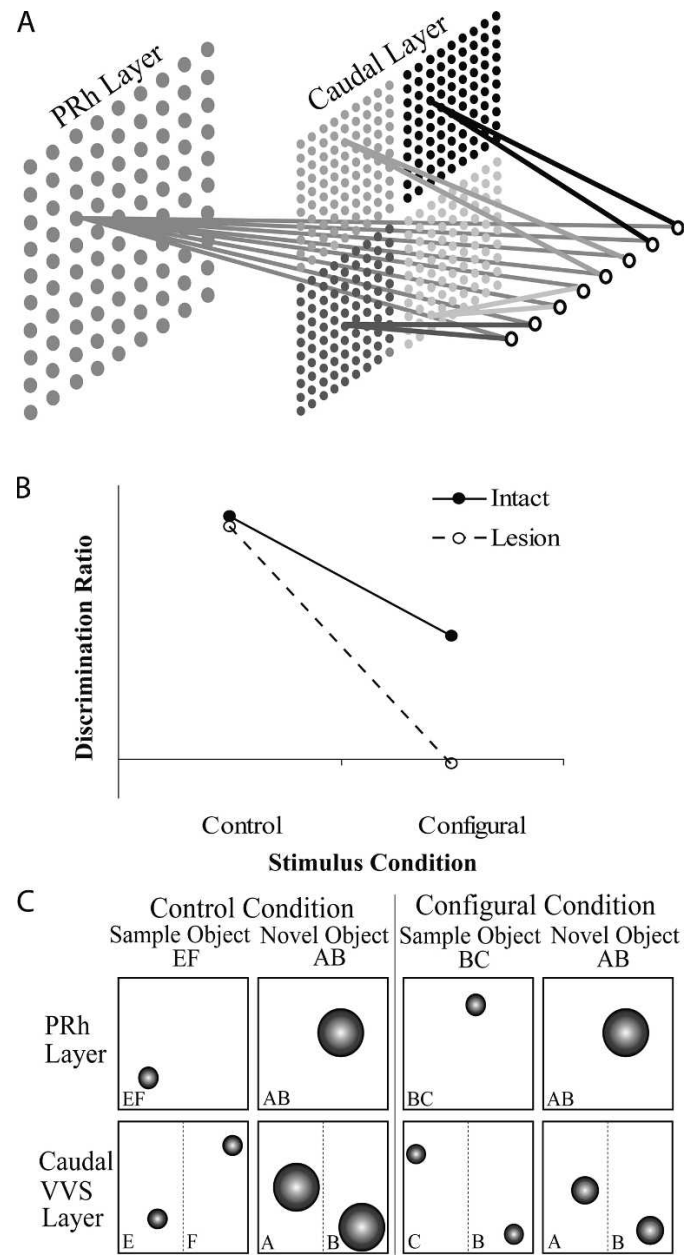

Figure 1. (A) Illustration of the connectionist model. The input layer, containing eight nodes, is shown on the far right; the two layers of stimulus representations (perirhinal cortex [PRh] and caudal) are shown to the left of the input layer. Stimulus inputs to the network have eight "stimulus dimensions" (attributes); each dimension is represented in the diagram by an individual input node. On the caudal layer, stimulus dimensions are paired into four simple conjunctions. Each simple conjunction is shown in a different shade of gray and is represented individually on the caudal layer. On the perirhinal cortex layer, the eight stimulus dimensions are combined into a conjunction, shown in gray, which represents the whole stimulus. ( $B$ ) Performance of the model during object recognition in two conditions, control and configural. Filled circles represent recognition of the control group, and open circles represent recognition scores of the lesion group. (C) Stimulus representations on the Kohonen grids of the model in the choice phase for the control (left) and configural (right) object recognition conditions. The PRh layer represents the object stimulus with a single conjunctive representation. The caudal layer represents individual object features separately; stimulus representations in the caudal layer are shown as chunked according to object "features," i.e., half an object stimulus. Small circles indicate sharply tuned ("familiar") representations, and large circles indicate coarsely tuned ("unfamiliar") representations. For discussion, see Results.

sharply tuned and can be discriminated from the coarsely tuned conjunctive representation of the novel stimulus. However, in the configural condition, the novel object is composed of features that have both been presented as part of one or other sample stimulus. This means that on the caudal layer of the model, both features possessed by the novel stimulus appear familiar since their representations have been tuned through encoding of the sample stimuli; the representations of the novel and sample stimuli on the caudal layer are therefore indiscrim- 
inable on the basis of familiarity. In contrast, on the PRh layer the representations of the whole conjunction are distinct for the familiar and novel stimuli, even though the individual features of the novel object have been presented to the network before, so that only the familiar representation is sharply tuned and therefore the novel and familiar objects are readily discriminable. It is clear from the schematic illustration of the model's representations shown in Figure $1 \mathrm{C}$ that removing the rostral (PRh) layer, leaving only the caudal layer to solve the discrimination, will result in a behavioral impairment.

\section{Experiment 2: Minimal-delay configural spontaneous object recognition}

Experiment 2 was designed to examine the role of PRh cortex in a configural spontaneous object recognition task. Rats with PPRh lesions and control rats were tested in two conditions, control and configural using a minimal delay between testing phases. The configural object recognition task was designed to test whether PPRh-lesioned rats could recognize a novel configuration of stimulus elements. In the configural condition of the object recognition task, the novel stimulus $(\mathrm{AB})$ was created from features of the compound stimuli presented in the two sample phases (BC and AD) (Fig. $2 \mathrm{~A})$. As a result, the rat was presented with one of the sample stimuli (BC) and a novel configuration of already seen, familiar features from $\mathrm{BC}$ and $\mathrm{AD}(\mathrm{AB})$ (see Fig. 2B). Thus, the novel object could not be identified on the basis of its features, only the conjunction of features was novel. In the control condition of the task, the novel stimulus $(\mathrm{AB})$ was created to share minimal features in common with the stimuli presented in the two sample phases (EF and GH). Thus, the novel feature could be identified on the basis of individual features. We predicted that PPRh-lesioned rats would be impaired in the configural (high feature ambiguity) condition of the object recognition task. Furthermore, since the novel stimulus in the control condition could be identified by features alone and did not require the rat to resolve conjunctions of features (low feature ambiguity), we predicted that rats with PPRh lesions would be able to discriminate the novel stimulus in the choice phase of the task and perform similarly to control rats.

\section{Histological analysis}

Throughout this study, histological assessment was made with reference to the anatomical designations of Burwell (2001). In the PPRh group, extensive cellular loss was revealed throughout the perirhinal and post-rhinal cortices (Fig. 3A). The lesion was observed in the rostral border of the PRh and continued caudally throughout perirhinal and post-rhinal cortices. The lesion also extended ventrally to include the lateral entorhinal cortex and the piriform cortex in all PPRh animals. There was some unilat- eral sparing of the most rostral PRh in three animals. Minor unilateral damage to area CA1 in the ventral hippocampus was observed in three animals. In two of the PRh animals, damage to the auditory association cortex, immediately rostral to TE, occurred. Unilateral cortical damage was seen in two PPRh rats, visible in the parietal cortex from $0.49-3.14 \mathrm{~mm}$ posterior to bregma. $\mathrm{Mi}$ nor, incidental TE damage was observed in all PPRh animals (bilateral in two PPRh rats and unilateral in three PPRH rats). However, comparison of the discrimination scores of PPRh rats with (unilateral or bilateral) TE damage revealed no significant differences $\left(F_{(1,3)}=0.65\right)$ and no interaction with condition $(F<1)$. Unilateral minor cortical damage was observed in two control rats, visible from $\sim 0.92-2.56 \mathrm{~mm}$ posterior to bregma in PPRh.

\section{Behavioral analysis}

Duration of the sample phase

All animals explored the sample stimuli for $25 \mathrm{sec}$ in under $5 \mathrm{~min}$ on all trials. The total time required to complete $25 \mathrm{sec}$ of exploration in each sample phase was averaged and analyzed since significant group differences at this stage of the trial might lead to differences in subsequent recognition performance. This analysis revealed no significant difference between the groups 

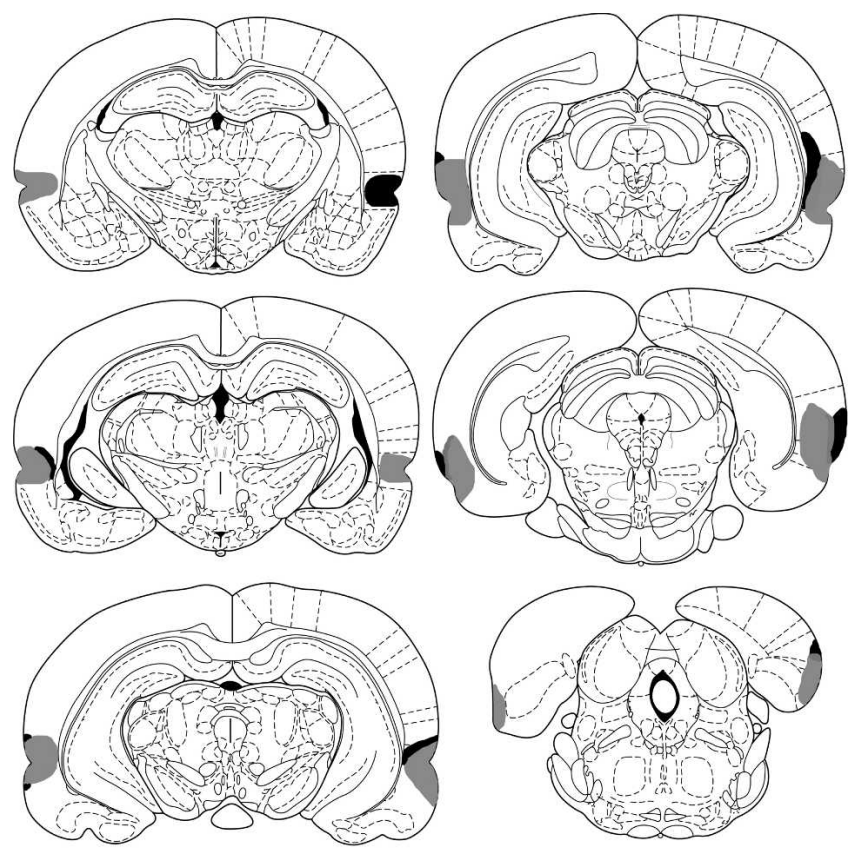

A

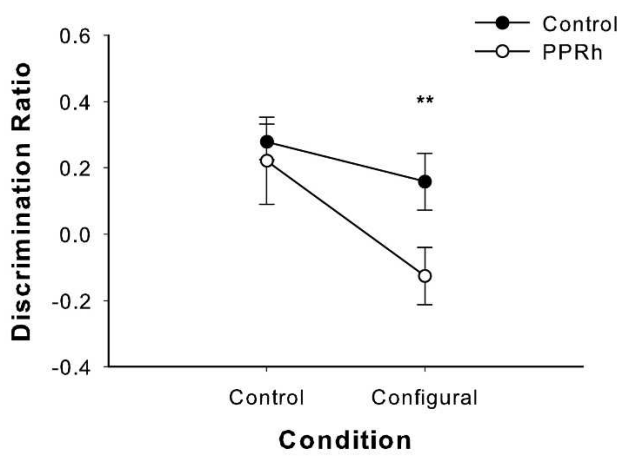

B

Figure 3. (A) Coronal sections illustrating the extent of the largest (gray) and smallest (black) lesions of the perirhinal and post-rhinal cortex in experiments 2 and 3 , from -3.14 to $-8.72 \mathrm{~mm}$ posterior to bregma. Reprinted from Paxinos and Watson (1998), with permission from Elsevier () 1998. (B) Performance on minimal delay configural object recognition (experiment 2). Data are presented as average discrimination ratio \pm SEM. ${ }^{*} P<0.05$.

$(F<1)$ and no significant effect of condition $(F<1)$. The interaction of group by condition was also not significant $(F<1)$. The mean sample phase duration $( \pm$ SEM) for groups in each condition was as follows: control condition: $\mathrm{PPRh}=153.71 \pm 14.25$ sec, control $=147.48 \pm 15.21 \mathrm{sec}$; configural condition: $\mathrm{PPRh}=143.90 \pm 16.30 \mathrm{sec}$, control $=140.74 \pm 17.70 \mathrm{sec}$.

Object exploration during the choice phase

Analysis of the total mean object exploration during the choice phase revealed no significant group effect $\left(F_{(1,10)}=1.09\right)$ and no significant effect of condition $\left(F_{(1,10)}=4.28\right)$. The interaction of group by condition was also not significant $(F<1)$. The mean choice exploration ( \pm SEM) for groups in each condition was as follows: control condition: $\mathrm{PPRh}=10.93 \pm 2.11 \mathrm{sec}$, control $=14.07 \pm 2.92 \mathrm{sec}$; configural condition: $\mathrm{PPRh}=14.50 \pm 2.0$ sec, control $=15.64 \pm 2.01 \mathrm{sec}$.

Recognition during the choice phase

The PPRh group performed significantly worse than the control group in the configural condition but not in the control condition (Fig. 3B). A two-way ANOVA with repeated measures conducted on the discrimination ratio revealed a significant main effect of group $\left(F_{(1,10)}=9.40, P<0.05\right)$ and condition $\left(F_{(1,10)}=5.49, P<0.05\right)$ but revealed no significant interaction of group by condition $\left(F_{(1,10)}=1.31\right)$. As we predicted an effect of group in the configural condition, but not in the control condition, planned comparison t-tests were used to analyze the discrimination ratio. This analysis revealed that the performance of PPRh rats in the control condition was not significantly different from control rats (control condition: $\mathrm{t}<1, P>0.05$ ) while performance of PPRh-lesioned rats in the configural condition was significantly worse than control rats (configural condition: $t$ $[10]=2.50, P<0.05)$. Thus, in the configural condition with a minimal delay between testing phases, PPRh-lesioned rats were unable to recognize the novel stimulus, which had familiar features but a novel configuration, necessitating the use of configural representations to facilitate recognition. When the novel stimulus in the choice phase did not contain familiar features from the sample stimuli and therefore could be recognized as novel on the basis of features alone, the PPRh and control group did not differ: Both groups could discriminate the novel from the familiar stimulus in the choice phase.

\section{Experiment 3: Zero-delay configural spontaneous object recognition}

In order to further reduce the mnemonic load in the task, the configural object recognition paradigm used in experiment 2 was refined to allow no delay between the two sample phases and the choice phase (a "zero-delay" condition) (Fig. 4A). In experiment 3 , we reduced the delay between testing phases to 0 sec in order to minimize the mnemonic demand of the object recognition task. The zero-delay condition in object recognition experiments is usually considered to have little or no memory load and has been used as an assay of perceptual function. The same rats from experiment 2 were tested in experiment 3 , using new objects but using the same protocol of testing as before, and there was no delay between testing phases. Performance in a zero-delay object recognition task is regarded as an index of perceptual (and not mnemonic) function (Eacott et al. 1994; Buffalo et al. 1999, 2000), as there is little or no long-term memory demand. We also wanted to examine whether changing the stimulus material from Lego objects to junk objects would replicate the impairment of PRh-lesioned rats in the configural object recognition task from experiment 2 . In addition, the use of junk objects sets the stage for comparison with the junk object oddity task in experiment 4 . As in experiment 2, the performance of PPRh-lesioned animals in the control condition was expected to be similar to control rats, and their performance in the configural condition was expected to be impaired.

\section{Histological analysis}

The same rats used in experiment 2 were used in experiment 3.

\section{Behavioral analysis}

\section{Duration of the sample phase}

All animals explored during the sample phases for $25 \mathrm{sec}$ in under $5 \mathrm{~min}$ on all trials. The total time required to complete $25 \mathrm{sec}$ of 


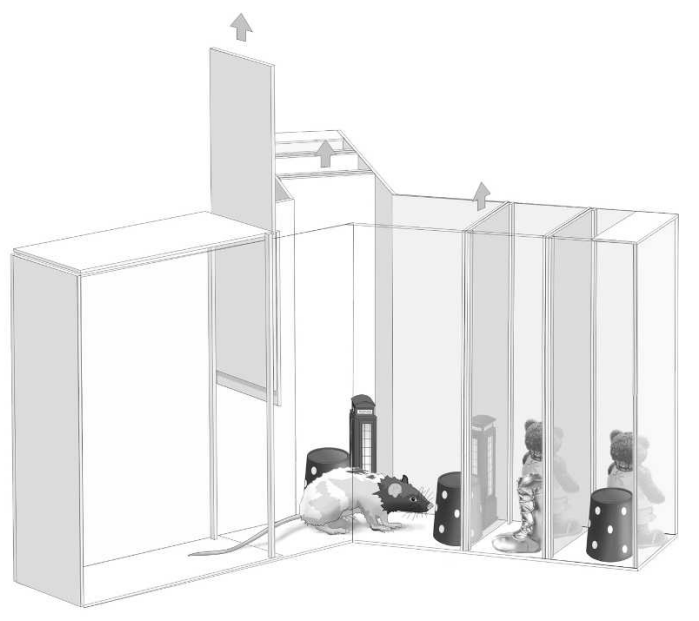

A

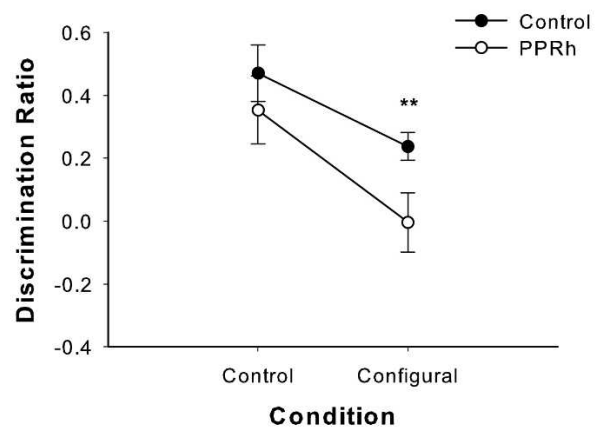

B

Figure 4. (A) Illustration of the apparatus and representative stimuli used in the zero-delay object recognition task in experiment 3 . The figure illustrates examples of stimuli that could appear in the configural condition during a given trial. The nearest wall appears transparent for illustrative purposes and the guillotine door is shown raised. The sample 1 objects are closest to the rat, the sample 2 objects are located behind the sample 1 objects, and the choice objects are the next set of objects behind the sample 2 objects. The door behind the choice objects remained closed during all testing. All stimuli (sample 1, sample 2, and choice objects) were placed in the apparatus before testing began. At the beginning of a trial, the rat was released from the start box when the experimenter raised the guillotine door. In sample phase 1, the rat was exposed to identical versions of the same object. At the end of the sample phase 1, the objects were removed, and the door between the sample 1 and sample 2 objects was immediately raised. In sample phase 2, the rat was exposed to different identical versions of the same object. At the end of the sample phase 2, the objects were removed, and the door between the sample 2 and choice objects was immediately raised. In the choice phase, the rat was exposed to a third, identical copy of the sample object $\mathrm{EF}$ at the end of the one exploration arm and a novel configuration Lego object (AB) at the end of the other arm. (B) Performance on zero-delay configural object recognition (experiment 3 ). Data are presented as average discrimination ratio \pm SEM. ${ }^{*} P<0.05$.

exploration in the sample phase was analyzed. This analysis revealed no significant difference between the groups $(F<1)$ and no significant effect of condition $(F<1)$. Furthermore, the interaction of group by condition was also not significant $(F<1)$. The mean sample phase duration $( \pm$ SEM) for groups in each condition was as follows: control condition: $\mathrm{PPRh}=141.53 \pm 12.70$ sec, control $=134.07 \pm 13.15 \mathrm{sec}$; configural condition: $\mathrm{PPRh}=136.73 \pm 38.10 \mathrm{sec}, \mathrm{control}=114.36 \pm 26.22 \mathrm{sec}$.

Object exploration during the choice phase

Analysis of the total mean object exploration during the choice phase revealed no significant group effect $\left(F_{(1,10)}=1.70\right)$. The PPRh and control groups combined explored the novel and familiar choice objects more in the control condition than in the configural condition $\left(F_{(1,10)}=7.61\right.$; control condition: $\mathrm{PPRh}=13.97 \pm 3.70 \mathrm{sec}$ and control $=11.84 \pm 1.40 \mathrm{sec}$; configural condition: $\mathrm{PPRh}=13.11 \pm 1.82$ sec and control $=8.34 \pm 3.30 \mathrm{sec}$ ). However, the interaction of group by condition was not significant $(\mathrm{F}<1)$.

\section{Recognition during the choice phase}

The PPRh-lesioned rats performed significantly worse than the control rats in the configural condition but not in the control condition (Fig. 4B). A two-way ANOVA with repeated measures conducted on the discrimination ratio revealed a significant main effect of group $\left(F_{(1,10)}=5.49, P<0.05\right)$ and condition $\left(F_{(1,10)}=14.69, P<0.01\right)$ but revealed no significant interaction of group by condition $(F<1)$. As we predicted an effect of group in the configural condition but not in the control condition, planned comparison t-tests were used to analyze the discrimination ratio. This analysis revealed that the performance of PPRh rats in the control condition (control: $\mathrm{t}<1, P>0.05$ ) was not significantly different from the control rats, while performance of PPRh-lesioned rats in the configural condition was significantly worse than control rats (configural: $\mathrm{t}[10]=2.83, P<0.05$ ). Thus, PPRh-lesioned rats were not impaired relative to the control group in the control (nonconfigural) condition but were significantly impaired when the novel object in the choice phase had familiar features but a novel configuration, replicating the PPRh-lesion induced deficit in experiment 2.

\section{Experiment 4: Configural oddity discrimination}

Impairments following lesions to PPRh were revealed in both the minimal and zero-delay configural object recognition task in experiments 2 and 3 using Lego objects and junk objects, respectively, and these deficits emerged only when the stimuli to be discriminated in the choice phase contained a novel configuration of already seen features from the sample phases. Although we used a zero-delay condition in experiment 3 , one could argue that the PPRh lesion-induced deficit emerged because of a failure in mnemonic processing and not a failure of the PPRh group to resolve feature ambiguity contained in the configural stimuli. Although there was no delay, rats had to remember the familiar objects from the sample phases during the time of the discrimination (in the choice phase) to discriminate the novel stimulus. Therefore, in experiment 4, we tested the effects of PRh lesions on a configural simultaneous oddity discrimination test (Fig. 5A), in which all objects were presented simultaneously and the mnemonic demand was therefore minimized or eliminated. Rats were presented with two copies of one object pair (control condition: EF 1 and EF 2; configural condition: CB 1 and CB 2), two copies of a different object pair (control condition: GH 1 and GH 2; configural condition: AD 1 and AD 2), and one copy of an "odd" object (control condition: $\mathrm{AB}$; configural condition: $\mathrm{AB}$ ). The odd object in the configural condition could only be identified by using the conjunction of features of the two different object pairs, while the odd object in the control condition could be identified by using elemental features alone. Therefore, five objects were presented simultaneously, an odd object and two different identical object pairs. We predicted that the rat would divide its exploration between the two identical objects within the pairs (control condition: EF 1 and EF 2, GH 1 and GH 2; 


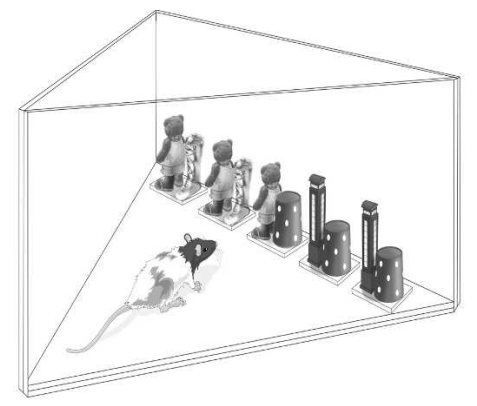

A

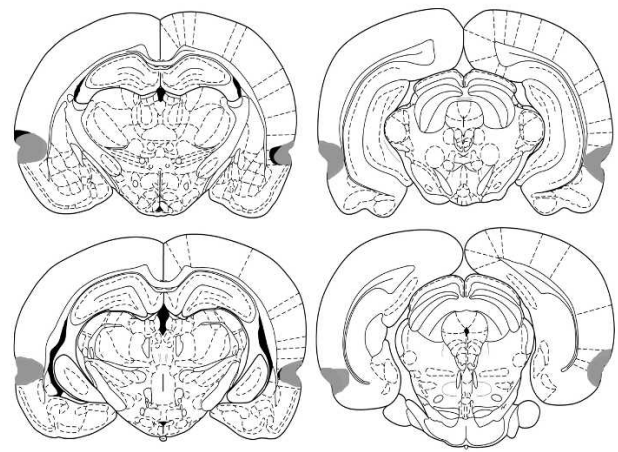

B
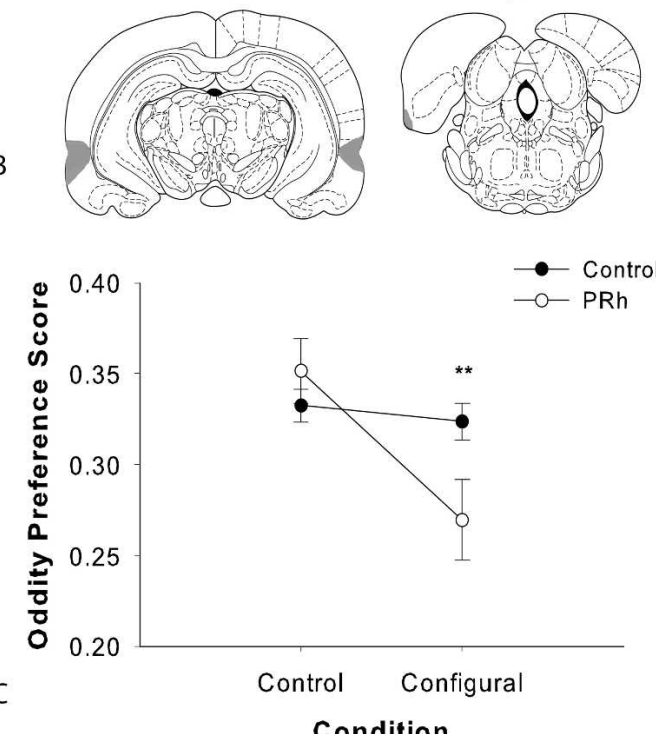

Condition

Figure 5. (A) The spontaneous oddity apparatus used in experiment 4. All stimuli were placed in the apparatus before testing began. Exploration of the objects was recorded by the experimenter. The odd object could appear in any of the five locations; here, it is shown in the center location and the configural condition is shown ( $A D 1, A D 2, A B, B C 1$, and $B C 2$ ). (B) Coronal sections illustration the extent of the largest (gray) and smallest (black) lesions of the perirhinal cortex in experiment 4 , from -3.14 to $-8.72 \mathrm{~mm}$ posterior to bregma. Reprinted from Paxinos and Watson (1998), with permission from Elsevier () 1998. (C) Spontaneous configural oddity preference by control and PRh groups in experiment 4 . Data are presented as 2-min cumulative average oddity preference scores (the exploration of the odd object divided by the total exploration of the odd and identical object pairs combined). \pm SEM. ${ }^{*} P<0.05$.

configural condition: $\mathrm{CB} 1$ and $\mathrm{CB} 2, \mathrm{AD} 1$ and $\mathrm{AD} 2$ ), resulting in an overall apparent "preference" for the odd object. An observed impairment in this task would extend the results found in experiments 2 and 3 and would further suggest that PRh is necessary for resolving feature conjunctions in complex stimuli.

\section{Histological analysis}

In the PRh group (Fig. 5B) from experiment 4, cellular loss was revealed throughout the $\mathrm{PRh}$. The lesion was confined to the $\mathrm{PRh}$ and extended approximately from $3.14-7.04 \mathrm{~mm}$ posterior to bregma in most animals. Minimal unilateral TE damage was observed in three of the PRh-lesioned animals. Comparison of the mean odd object preference scores between PRh rats with unilateral PRh damage and rats with no damage to area TE showed no significant effect of group $\left(F_{(1,10)}=1.73\right)$ and no interaction with condition $\left(F_{(1,10)}=1.60\right)$. There was some unilateral sparing of the most rostral PRh in four animals. Two PRh-lesioned rats did not incur substantial damage to the PRh, and their data were omitted from statistical analyses. Unilateral cortical damage was seen in one PRh rat and two control rats, visible in the parietal cortex from $0.92-2.56 \mathrm{~mm}$ posterior to bregma. The damage is possibly a result of the craniotomies or from inserting the needle.

\section{Behavioral analysis}

Object exploration during the oddity task

Total exploration of the five objects during the oddity task was not affected by lesion. ANOVA revealed no significant interaction of group by condition $\left(F_{(1,24)}=3.90\right)$, and no main effects of group $(F<1)$ or condition $(F<1)$. The mean exploration during the oddity discrimination task $( \pm$ SEM) for each group in each condition was as follows: configural condition: control $=39.00 \pm 5.15 \mathrm{sec}$ and $\mathrm{PRh}=40.15 \pm 6.30 \mathrm{sec}$; control condition: control $=42.01 \pm 5.07 \mathrm{sec}$ and $\mathrm{PRh}=41.30 \pm 4.73 \mathrm{sec}$.

\section{Preference for the odd object}

The odd object preference score for each group was analyzed for the second minute of exploration during the oddity task, since this was the first time point at which the control group showed a significant preference for the odd object in both conditions. Analysis of the odd object preference score at 2 min revealed a significant main effect of condition $\left(F_{(1,24)}=11.50, P=0.002\right)$, and the interaction of group by condition was also significant $\left(F_{(1,24)}=8.32, P=0.008\right)$ (Fig. 5C). However, there was no significant group effect $\left(F_{(1,24)}=1.42\right)$. We predicted an effect in the configural condition but not the control condition, and planned comparisons of condition with independent samples t-tests found no significant difference between the groups in the control condition (t $[24]=1.75, P>0.05$ ); however, the PRh group was significantly impaired relative to the control group in the configural condition (t $[24]=3.30, P<0.05)$.

\section{Discussion}

The present results provide evidence that PRh is critical for resolving feature ambiguity in explicitly configural spontaneous object recognition and simultaneous oddity discrimination tasks. Configural manipulations, which produce high levels of feature ambiguity, have been shown to impair performance of animals and humans with PRh damage on two-choice visual discrimination tasks. The present study is, to our knowledge, the first to show that PRh resolves feature ambiguity in configural object recognition or oddity tasks. Therefore, the deficits observed following PRh lesions in both object recognition and oddity discriminations bring together the results of previous studies that have shown that PRh is needed for visual discrimination and recognition memory tasks. The common factor that led to impairments in all of these tasks is feature ambiguity, regardless of whether the task was perceptual or mnemonic.

We have shown that, as predicted and as made explicit by the simulations in experiment 1 , PPRh lesions can produce performance deficits in a configural version of spontaneous object 
recognition using a minimal delay between testing phases (experiment 2). Furthermore, PPRh lesions can produce performance deficits in the same configural object recognition task when the mnemonic demands of the task are further minimized in a zero-delay condition (experiment 3). Finally, in experiment 4 we examined the performance of rats with selective PRh lesions and control rats in the configural simultaneous oddity discrimination task. When configural stimuli were presented simultaneously, such that there was little in the way of long-term memory demands, PRh lesions still produced profound impairments. The failure of the PRh group to show preference for the odd object can be viewed as an inability to resolve feature ambiguity arising from the configural arrangement of stimuli. Because the oddity task does not require the rat to remember information across a delay, this latter result provides further evidence that PRh is not only important for memory but also for the perceptual discrimination of complex stimuli under conditions of high feature ambiguity.

Norman and Eacott (2004) have reported object recognition deficits following manipulations of what might be called "spatial feature ambiguity." Feature ambiguity was created by presenting a spatially reconfigured sample stimulus as the novel stimulus. Since feature ambiguity is described by the Perceptual Mnemonic Feature Conjunction model as a quality that emerges when perceptual similarities are shared between stimuli, it does not account for feature ambiguity in spatial reconfigurations (Bussey et al. 2002). However, it is unclear whether the deficit following PRh lesions revealed in Norman and Eacott (2004) was the result of purely spatial feature ambiguity. When the novel spatial rearrangement of a familiar stimulus was created, some new features were exposed while other features were concealed, thus creating nonspatial feature ambiguity. As a result, this manipulation may be similar to the feature ambiguous "morph" manipulation used by Bussey et al. (2003), in which certain features were "faded in" to a stimulus and others were "faded out." This manipulation led to impairments in monkeys with PRh lesions (Bussey et al. 2003). Since PRh lesions can have little effect on spatial tasks (Glenn and Mumby 1998; Bussey et al. 1999, 2000) and c-fos studies indicate that PRh is not activated by spatial rearrangements of objects (Wan et al. 1999; Jenkins et al. 2004), we favor the idea that $\mathrm{PRh}$ is involved specifically in nonspatial feature ambiguity.

These same investigators have also previously examined PRh-lesioned rats in a spontaneous "object-in-context" recognition task (Norman and Eacott 2005). During this task, a configuration of an object and a location was presented as the novel stimulus in an open field. This task could be viewed as a version of the biconditional task (M.J. Eacott, pers. comm.). Identical objects A were paired with context a in the first exposure phase (Aa and $\mathrm{Aa}$ ), identical objects $\mathrm{B}$ were paired with context $\mathrm{b}(\mathrm{Bb})$ in the second exposure phase ( $\mathrm{Bb}$ and $\mathrm{Bb}$ ), and a third copy of the configuration $\mathrm{Aa}$ and the novel configuration $\mathrm{Ab}$ was presented in the choice phase. Therefore, similar to the configural object recognition task used in the present study, only the novel configuration-in this case of object and context, and not the individual objects and contexts-was novel in the choice phase. During this task, both PPRh and PRh lesions produced significant recognition impairments at both a 2-min and a 5-min delay.

The present study complements a previous study by Bartko et al. (2007). In that study, we showed that PRh lesions led to impairments in object recognition and oddity tasks when stimuli were perceptually similar but not when they were perceptually dissimilar. The finding that perceptual manipulations adversely affecting subjects with PRh lesions in two-choice discrimination tasks (Bussey et al. 2002, 2003) also adversely affect PPRh and
PRh-lesioned rats in object recognition and oddity tasks suggests that a common mechanism may underlie PRh involvement in visual discrimination and recognition tasks.

Similarly, in the present study, configural manipulations in object recognition and oddity tasks led to substantial impairments in animals with damage to PRh. The finding that configural manipulations that adversely affect subjects with PRh lesions in visual discrimination tasks (Buckley and Gaffan 1998; Eacott et al. 2001; Bussey et al. 2003; Saksida et al. 2007) also adversely affect PRh and PPRh-lesioned rats in object recognition and oddity tasks also provides additional evidence that a common mechanism underlies the involvement of PRh in visual discrimination and recognition tasks.

We have suggested that the underlying explanation for PRh lesion-induced impairments on all of these tasks is that PRh contains conjunctive representations for the resolution of feature ambiguity. Feature ambiguity is high in configural tasks and in certain perceptual tasks in which stimuli are made more similar by, for example, morphing. Simulations using computational models have accounted for PRh lesion-induced impairments after both of these types of manipulations, in both two-choice discrimination tasks and object recognition tasks (Bussey and Saksida 2002; Bussey et al. 2002, 2003; Cowell et al. 2006; Bartko et al. 2007), illustrating explicitly how the resolution of feature ambiguity by complex conjunctive representations in PRh can explain all of these findings. The oddity task in the present study provides a good illustration of how both configural tasks and perceptually difficult discriminations can be thought of as feature-ambiguous tasks. Consider the array of objects used in the oddity task shown in Figure 5B. On one hand, we can describe the task in configural terms, as follows: The features (elements is an equivalent term used by configural theorists) (Sutherland and Rudy 1989; Pearce 1994) in the odd object are present in other objects in the array; as a result, the features are ambiguous and the task cannot be solved on the basis of the features alone. However, the configuration of features that identifies the odd object is not found in any other object. Thus in control subjects, the conjunctive or configural representation in PRh resolves the feature ambiguity in the task, and the odd object can be identified as such. Alternatively, we can describe the task in perceptual terms: In Figure 5A, the odd object is perceptually similar to the two objects to its left (half of their features are shared). The odd object is also perceptually similar to the two objects to its right. Thus the task requires the subject perceptually to discriminate the odd object from the other objects in the array. But this, too, is feature ambiguity: The objects cannot all be discriminated from each other on the basis of simple features alone; only the conjunction of features makes the odd object perceptually unique. This analysis can be extended to standard configural tasks such as the biconditional task, in which learning takes place slowly across trials. This, too, is a task where items must be discriminated perceptually from each other but where the "perceptual" similarity between the stored representations of the items is revealed through stimulus sampling that occurs not at once, as in the oddity task, but across trials. The perceptual similarity between items - the feature ambiguity-builds up across trials. This analysis may seem odd, as configural tasks such as the biconditional task have been used solely in the context of the study of learning and memory systems (Rudy and Sutherland 1989; Alvarado and Rudy 1992, 1995). But our analysis of the functions of PRh, culminating in the results of the present study, has led us to propose that there is, in fact, no neat division of "perception" and "memory" into discrete modules in the brain (Murray et al. 2007), and so, perception and memory need not be considered independently. Instead, the ventral visual-PRh-hippocampal stream can be thought of as a hierarchically organized represen- 
tational continuum that mediates both perception and memory (Bussey and Saksida 2005, 2007).

\section{Materials and Methods}

\section{Experiment 1}

\section{Architecture of the model}

This section provides a brief overview of the connectionist network (see Fig. 1A; for details, see Cowell et al. 2006). The model assumes that regions of the ventral visual stream, including PRh, contain visual representations that are organized hierarchically, with simple features being housed in caudal regions of the ventral visual stream, and representations of the conjunctions of those features residing in more rostral regions (Bussey et al. 2002). In the connectionist network, this system of representations is reduced to a two-stage scheme, in which the first layer corresponds to a caudal region of the ventral visual stream, and the second layer to PRh.

The caudal layer of the model combines two stimulus dimensions into single representations. The PRh layer combines eight stimulus dimensions into a single representation, forming a unique and fully specified representation of a visual object. Both layers of the model are implemented using Kohonen grids. The caudal layer comprises four Kohonen grids, each of which receives two-dimensional inputs, and the PRh layer comprises one Kohonen grid receiving an eight-dimensional input. Thus, a given stimulus is represented as a single complex conjunction on the PRh layer and as a series of separate, two-dimensional conjunctions in the caudal layer.

Kohonen grids are designed to model cortex, including computational abstractions of cortical mechanisms such as lateral inhibition; this type of network is therefore appropriate for the current investigation. Each Kohonen grid comprises a twodimensional array of processing units that receives stimulus inputs and is characterized by lateral inhibitory feedback between neighboring units. The grids are trained by the successive presentation of a number of stimulus inputs; the weights of each unit are incrementally adapted on each presentation. This results in an automatic mapping of stimulus inputs onto a set of representations that possess the same topological order as the stimuli, that is, similar stimuli are represented in neighboring locations on the grid. The self-organization process involves the sharpening of representations of stimuli on which the network is trained. A novel stimulus will elicit a moderate level of activity, broadly distributed across a large number of units in the grid (top panel of Figure 3 in Cowell et al. 2006); as that stimulus is presented repeatedly, the activation pattern it elicits becomes more selective until only a small area of the grid contains highly active units, producing a peak of activation (bottom panel of Figure 3 in Cowell et al. 2006). The development of sharply tuned representations thus can be used as the basis for familiarity judgments: As a stimulus representation becomes sharper, so it is judged to be more familiar (Norman and O'Reilly 2003).

\section{Simulation methods}

\section{Stimuli}

Object stimuli in this experiment were created by constructing whole objects from a pool of visual attributes, or stimulus dimensions. Each object comprised eight stimulus dimensions in total. On the caudal layer, objects are represented as a series of separate, low-dimensional conjunctions of stimulus dimensions to reflect the lesser complexity of individual visual representations coded for by neural firing in caudal VVS. In the present experiment and those that follow, object stimuli presented to networks and animals were compound stimuli possessing two components, which we have termed object "features." In the model, a whole object is represented on the caudal layer by four simple conjunctions of two dimensions, meaning that each object featurecorresponding to half an object stimulus-is represented by two simple conjunctive representations on the caudal layer. On the PRh layer, a whole object comprising eight dimensions is repre- sented as a single complex conjunction. Real-world objects may be thought to contain more stimulus dimensions than this, but the model is designed to illustrate a principle rather than reproduce the real-world situation strictly veridically. Cowell et al. (2006) provides details of the architecture of the model and how stimuli are represented in the two layers.

Eight sets of stimuli were created for the present experiment; each set comprised two sample objects (sample 1 and sample 2) and a novel object $(\mathrm{N})$. Four of the eight stimulus sets were assigned to the "control" condition, and the other four sets were assigned to the "configural" condition. In the control stimulus sets, no features were shared by sample 1 , sample 2 , and novel, such that the novel object presented at the choice phase was composed of two entirely novel features. In the configural stimulus sets, the novel stimulus was composed of one feature that had appeared in sample 1 and one feature that had appeared in sample 2, such that both features that the novel object possessed were familiar and only the conjunction of features was novel. Neither of the two sample stimuli nor the novel stimulus in any set was replicated in any other set.

\section{Simulation procedure}

Two groups of six networks were tested: Group Intact consisted of intact networks, and Group Lesion consisted of networks in which the PRh layer had been removed to simulate PRh lesions. Each network was tested on four object sets under each condition-control and configural-giving eight trials per network. Networks were initialized and pretrained before testing on the four trials (for details, see Appendix 1 of Cowell et al. 2006). On each trial, a network was presented with the first sample object and allowed to "encode" the object for 20 cycles; each cycle sharpened incrementally the peak of activation representing the sample object. Next, the network was presented with the second sample object and allowed to encode that for 20 cycles. Following encoding of both samples, the network was presented with one of the sample objects (sample 1 in half of the trials and sample 2 in the remaining trials) and the novel object in a "choice" phase. Whether the sample object presented during the choice phase had previously been presented as sample 1 or sample 2 was counterbalanced within and across groups of networks and across stimulus sets. No learning occurred in the choice phase; the representations of the two objects were simply assessed to obtain an index of their relative familiarity (i.e., the recognition score in the present article). For derivation of the recognition score from the activation patterns elicited by the sample and novel stimuli, see Appendix 1 of Cowell et al. (2006). At the beginning of every new trial, each network was reset to the state it had assumed at the end of pretraining.

\section{Experiment 2}

\section{Subjects}

The subjects were 12 adult male Lister hooded rats weighing 270$320 \mathrm{~g}$ before surgery and housed in pairs in a room with a $12 \mathrm{~h}$ light/dark cycle (lights on at 7:00 p.m.). All behavioral testing was conducted during the dark phase of the cycle. During testing, rats were fed $\sim 15 \mathrm{~g}$ of laboratory chow after daily behavioral sessions to maintain weights at $85 \%-90 \%$ of free-feeding body weight. Water was available ad libitum throughout the experiment. All experimentation was conducted in accordance with the United Kingdom Animals (Scientific Procedures) Act (1986).

\section{Surgery}

Rats were divided into two groups: PPRh $(\mathrm{n}=5)$ and surgical controls (control; $\mathrm{n}=7$ ). Before surgery, all animals were deeply anesthetized by intraperitoneal injection $(60 \mathrm{mg} / \mathrm{kg}$, i.p.) of sodium pentobarbital and placed in a stereotaxic frame with the incisor bar set at +5.0 . The scalp was cut and retracted to expose the skull. Craniotomies were then performed directly above the target region, and the dura was cut to expose the cortex.

For the PPRh lesions, injections of $0.2 \mu \mathrm{L}$ of $0.9 \mathrm{M}$ NMDA dissolved in phosphate buffer $(\mathrm{pH} 7.4)$ were made through a 1- $\mu \mathrm{L}$ 
Hamilton syringe into five sites in each hemisphere. Each injection was made gradually over a 2-min period, and the needle was left in situ for an additional 4 min before being withdrawn. The stereotaxic coordinates relative to ear-bar zero were as follows: anteroposterior $(\mathrm{AP})+3.9$, lateral $(\mathrm{L}) \pm 5.9$, dorsoventral $(\mathrm{DV})$ +2.0; $\mathrm{AP}+2.4, \mathrm{~L} \pm 6.1, \mathrm{DV}+1.6 ; \mathrm{AP}+0.6, \mathrm{~L} \pm 6.2, \mathrm{DV}+2.5 ; \mathrm{AP}$ $-0.8, \mathrm{~L} \pm 6.2, \mathrm{DV}+2.7$; and $\mathrm{AP}-0.8, \mathrm{~L} \pm 6.2$, DV +4.3.

Control animals received sham PPRh surgeries. For sham surgeries, the same initial surgery was performed (including craniotomy and insertion of needle), but no injections were made. At the completion of surgery, the skin was sutured, and an antibiotic powder was applied. Animals were then administered subcutaneously with $5 \mathrm{~mL}$ of glucose saline.

\section{Histology}

After behavioral testing, rats were anesthetized by intraperitoneal injection of $2 \mathrm{~mL}$ of Euthatal (Rhône Mérieux) and perfused transcardially with $100 \mathrm{~mL}$ of PBS (pH 7.4), followed by $250 \mathrm{~mL}$ of $4 \%$ paraformaldehyde (PFA) pH 7.4. The brains were removed, post-fixed in $4 \% \mathrm{PFA}$ for $24 \mathrm{~h}$ at $4^{\circ} \mathrm{C}$, and then immersed in $25 \%$ sucrose in PBS until they sank. Coronal sections $(60 \mu \mathrm{m})$ were cut on a freezing microtome through the extent of the lesioned area, and every fifth section was mounted on a gelatin-coated glass slide, and stained with cresyl violet. Slides were examined under a light microscope to determine the extent of excitotoxininduced damage.

\section{Minimal delay configural spontaneous object recognition}

\section{Apparatus}

Spontaneous object recognition was conducted in a Y-shaped apparatus, as described previously (Winters et al. 2004; Forwood et al. 2005). Briefly, the Y-apparatus had high, homogenous white walls constructed from Perspex (Lucite International) to prevent the rat from looking out into the room, thereby maximizing attention to the stimuli. All walls were $40 \mathrm{~cm}$ high, and each arm was $27 \mathrm{~cm}$ in length and $10 \mathrm{~cm}$ wide. The start arm contained a guillotine door $18 \mathrm{~cm}$ from the rear of the arm. This provided a start box area within which the rat could be confined at the start of a given trial. The floor and walls were wiped down with a dry paper towel between trials but otherwise were not cleaned during the experiment. A lamp illuminated the apparatus, and a white shelf, $50 \mathrm{~cm}$ from the top of the apparatus, created a ceiling on which a video camera was mounted to record trials.

\section{General procedure}

All rats were habituated in two consecutive daily sessions in which they were allowed to explore the empty Y-apparatus for 5 min. For these habituation sessions, the rat was placed in the start box, and the guillotine door was opened to allow the rat to explore the main area of the apparatus. The guillotine door was lowered when the rat exited the start box to prevent re-entry into this area of the apparatus. The experimenter did not begin timing the trial until the rat exited the start box. Testing began $24 \mathrm{~h}$ after the second habituation session. Rats were given a series of test trials (one per day) with a minimum interval of $24 \mathrm{~h}$ between trials. A different object trio was used for each trial for a given animal, and the order of exposure to object pairs as well as the designated sample and novel objects for each trio were counterbalanced within and across groups. The time spent exploring objects was assessed from video recordings of the samples and choice phases. Data were collected by scoring exploratory bouts using a personal computer running a program written in Visual Basic 6.0 (Microsoft).

\section{Lego stimuli}

In the configural object recognition experiment, compound stimuli were used. A compound stimulus (e.g., AD) was created from two "features" (e.g., A and D) that were placed side by side. In each experiment, eight sets of stimuli, four configural sets and four control sets, were utilized. The features used in the configural sets can be represented generically as A, B, C, and D. These features were used to compose the configural sample stimuli $\mathrm{AD}$ and $\mathrm{BC}$ and the choice stimulus $\mathrm{AB}$ (see Fig. 2A). The features used in the control sets can be represented as E, F, G, H, A, and B. These features were used to compose the control sample stimuli $\mathrm{EF}$ and $\mathrm{GH}$ and the choice stimulus $\mathrm{AB}$ (see Fig. 2B). Each stimulus set contained triplicate $B C$, duplicate $A D$, and single $A B$ for the configural stimulus sets and triplicate $\mathrm{EF}$, duplicate $\mathrm{GH}$, and single $A B$ for the configural stimulus sets. Choice stimulus $A B$ in the control and configural condition was not the same object.

The Lego stimuli used in experiment 2 were composed entirely from Lego (LEGO group). Two Lego features (each feature was $3.3-15.6 \mathrm{~cm}$ tall and $1.9-2.3 \mathrm{~cm}$ wide) were affixed side by side to an $8.50 \times 8.50$-cm black Lego sheet. Lego objects were secured to the floor of the apparatus with Blu-Tack (Bostik). As far as could be determined, the Lego stimuli had no natural significance for the rats, and they had never been associated with a reinforcer.

\section{Minimal delay configural object recognition test}

Each test session consisted of three phases: sample 1, sample 2, and a choice phase. Duplicate sample 1 objects were placed in the apparatus before testing began. The rat was then placed in the start box with the guillotine door lowered. The guillotine door was then raised to allow the rat into the exploration area of the apparatus. When the rat exited the start box, the guillotine door was lowered to prevent re-entry, and the test phases began. When the rat exited the start box, the duplicate sample 1 objects were revealed to the rat. Sample 1 ended when the rat had explored the identical objects for $25 \mathrm{sec}$. At the end of sample 1, the rat was returned to the start box, and the duplicate sample 1 objects were removed and replaced with duplicate sample 2 objects; the guillotine door was opened, and the sample 2 objects were revealed to the rat. Sample 2 ended when the rat had explored the sample 2 objects for $25 \mathrm{sec}$. At the end of sample 2, the rat was returned to the start box, and the duplicate sample 2 objects were removed and replaced with the choice objects; the guillotine door was opened, and the choice objects were revealed to the rat. The choice phase contained an identical copy of the sample (familiar) object in one arm and a novel object in the other. The arm in which the novel object was placed was counterbalanced between rats and across trials. The time spent exploring the two objects in a testing phase was scored by an experimenter viewing the rat on a video screen. The cumulative duration of exploratory bouts, the beginning and end of which were indicated by pressing a given key on the computer keyboard, was calculated by the computer program. Exploration of an object was defined as directing the nose to the object at a distance of $<2$ $\mathrm{cm}$ and/or touching it with the nose.

The time spent exploring the novel and familiar objects was recorded for $3 \mathrm{~min}$ of the choice phase, but attention was focused on the first minute, during which object discrimination is typically greatest (Dix and Aggleton 1999). We calculated a discrimination ratio, the proportion of total exploration time spent exploring the novel object (i.e., the difference in time spent exploring the novel and familiar objects divided by the total time spent exploring the objects), for the first minute of the choice phase on each object recognition trial. This measure takes into account individual differences in the total amount of exploration time. The arm in which the novel object was placed was counterbalanced between rats and across trials.

Rats were tested in two conditions, control and configural; the presentation order of the two conditions was counterbalanced between rats and across trials. The novel stimulus position (right or left arm) was counterbalanced for the choice phase of the control and configural conditions. All rats received eight counterbalanced trials, four configural and four control trials. Each trial used a different set of objects. There was a minimum of $24 \mathrm{~h}$ between testing sessions.

\section{Control condition}

In the control condition, two identical Lego objects (EF 1 and EF 2 ) were presented in one sample phase (counterbalanced within and between groups as either sample 1 or sample 2 ), and the two 
identical Lego objects (GH 1 and GH 2) were presented in the other sample phase (counterbalanced within and between groups as either sample 1 or sample 2). During the choice phase, the apparatus contained an identical copy of the sample (familiar) object (EF 3) in one arm and a novel Lego object $(\mathrm{AB})$ in the other arm. As the features of this object set were novel, novelty could be judged on the basis of features alone.

\section{Configural condition}

In the configural condition, two identical Lego objects (BC 1 and BC 2) were presented in one sample phase (counterbalanced within and between groups as either sample 1 or sample 2), and the two identical Lego objects (AD 1 and AD 2) were presented in the other sample phase (counterbalanced within and between groups as either sample 1 or sample 2). During the choice phase, the apparatus contained an identical copy of the sample (familiar) object (BC 3) in one arm and a new Lego object (AB) in the other arm. As the features of the novel object were both familiar but the conjunction of features was novel, a judgment of novelty could be made on the basis of conjunction of features only and not on the basis of features alone.

\section{Data analysis}

Group means of three measures taken from object recognition testing (duration of sample phases, i.e., the time taken to accumulate criterion levels of exploration in the sample phases; total exploration time in the choice phase; and the discrimination ratio) were analyzed. Means from each of the three measures were submitted to a $2 \times 2$ ANOVA where the first factor was the between-subjects factor of lesion group and the second factor was the within-subjects factor of condition. Planned comparison $t$ tests were used when hypotheses predicted an effect of group in one trial condition but not the other. Significant interaction effects were further analyzed with independent samples t-tests. All tests of significance were performed at $\alpha=0.05$.

\section{Experiment 3}

\section{Subjects}

Twelve Lister hooded rats (the same rats used in experiment 2) were used for experiment 3.

\section{Zero-delay configural spontaneous object recognition}

\section{Apparatus}

To facilitate immediate viewing between test phases (a zero-delay condition), modifications were made to the original Y-apparatus described previously. Eight metal posts (two per arm, each 33.40 $\mathrm{cm}$ in height) were inserted and positioned $12 \mathrm{~cm}$ apart from each other, which in turn created holders for two sliding doors in each arm of the Y-apparatus. The doors (composed of white Perspex) were $33 \mathrm{~cm}$ tall and $10 \mathrm{~cm}$ wide.

\section{Junk object stimuli}

The construction of the stimuli was identical to experiment 2 . However, new junk object stimuli were used for experiment 3. The junk object stimuli were made of glass, plastic, or metal. Compound stimuli were used instead of single stimuli in order to create configural stimuli. Two junk object features (each feature was $2.50-20.0 \mathrm{~cm}$ tall and $1.80-7.50 \mathrm{~cm}$ wide) were glued to a Foamalux insert $(8.50 \mathrm{~cm} \times 9.60 \mathrm{~cm})$; objects were left to dry for several days. The insert containing the objects was secured to the floor of the apparatus with Blu-Tack. As far as could be determined, the junk objects had no natural significance for the rats, and they had never been associated with a reinforcer.

\section{Zero-delay configural object recognition test}

The general procedure of habituation, testing, and scoring was identical to experiment 2 . The zero-delay configural object recognition task was identical to the minimal delay configural object recognition task except instead of returning the rat to the start box after each test phase, the stimuli were removed immediately following a testing phase, and the doors between a given testing phase were immediately raised to expose the next stimuli for immediate viewing. Therefore, upon completion of exploration of sample 1 stimuli, the sample 1 stimuli were removed, the door was opened between sample 1 and sample 2 , and the rat was immediately shown the sample 2 stimuli. Upon completing sample 2, the stimuli were removed, the door between sample 2 and choice was opened, and the novel and familiar stimuli were immediately presented to the rat in the choice phase. All object sets used in a given trial were placed in the apparatus before the rat was placed in the start box.

Rats were tested in two conditions, control and configural as described in experiment 2 ; the presentation order of the two conditions was counterbalanced between rats and across trials. The novel stimulus position (right or left arm) was counterbalanced for the choice phase of the control and configural conditions. All rats received eight counterbalanced trials, four configural and four control trials. There was a minimum of $24 \mathrm{~h}$ between testing sessions.

\section{Data analysis}

The same data analyses were performed for experiment 3 that were performed for experiment 2 .

\section{Experiment 4}

\section{Subjects}

Twenty-eight experimentally naive Lister hooded rats (weighing 270-320 g) were used in experiment 4 . The animals were housed and fed in the same manner as the rats used in experiments 2 and 3.

\section{Surgery}

Rats were dived into two groups: PRh lesions $(\mathrm{n}=14)$ and surgical controls (control; $n=14$ ). Surgeries for the PRh group were identical to those in experiment 2 except that no needle was inserted into the lesion site for the control group (only a craniotomy was performed).

For the PRh lesions, injections of $0.2 \mu \mathrm{L}$ of $0.9 \mathrm{M}$ NMDA (Sigma) dissolved in phosphate buffer ( $\mathrm{pH} 7.4)$, were made through a $1-\mu \mathrm{L}$ Hamilton syringe into three sites in each hemisphere. Each injection was made gradually over a 2-min period, and the needle was left in situ for an additional 4 min before being withdrawn. The stereotaxic coordinates relative to ear-bar zero were $\mathrm{AP}+3.9, \mathrm{~L} \pm 5.9, \mathrm{DV}+2.0$; $\mathrm{AP}+2.4, \mathrm{~L} \pm 6.1, \mathrm{DV}+1.6$; and $\mathrm{AP}+0.6, \mathrm{~L} \pm 6.2, \mathrm{DV}+2.5$.

\section{Histology}

The same histological protocol was used as described previously in experiment 2.

\section{Configural oddity discrimination}

\section{Apparatus}

The oddity apparatus incorporated the same considerations used to design the Y-apparatus. The exploration area was triangular in shape (Fig. 5B). The oddity apparatus had high, homogenous white walls constructed from Perspex to prevent the rat from looking out into the room. All walls were $30 \mathrm{~cm}$ high and the three sides of the triangular area were $75 \mathrm{~cm}$ long. The back wall was $84 \mathrm{~cm}$ wide and $59 \mathrm{~cm}$ tall, and the objects were placed 2.50 $\mathrm{cm}$ apart along the back wall.

\section{Junk object stimuli}

Two of the stimulus sets from the control condition and two stimulus sets from the configural condition from experiment 3 were used as stimuli in experiment 4 . However, instead of using stimulus pair $\mathrm{BC}$, we rearranged the pair as $\mathrm{CB}$. This was to minimize any potential spatial component to the task. Two new sets of stimuli for each of the control and configural conditions were constructed for experiment 2. For the four new sets of stimuli (two configural sets and two control sets), two junk object features (each feature was $3.50-11.80 \mathrm{~cm}$ tall and $1.50-5.50 \mathrm{~cm}$ wide) were affixed an $8.50 \times 9.60 \mathrm{~cm}$ Foamalux insert. There- 
fore, eight sets of stimuli, four configural sets and four control sets, were utilized. The configural sets of stimuli contained duplicate $\mathrm{AD}$, duplicate $\mathrm{CB}$, and novel stimulus $\mathrm{AB}$. Each control sets of stimuli contained duplicate $\mathrm{EF}$, duplicate $\mathrm{GH}$, and novel stimulus $\mathrm{AB}$. In the control condition, objects $\mathrm{EF}, \mathrm{EF}, \mathrm{GH}, \mathrm{GH}$, and IJ were presented to the rat simultaneously. In the configural condition, objects $\mathrm{CB}, \mathrm{CB}, \mathrm{AD}, \mathrm{AD}$, and $\mathrm{AB}$ were presented to the rat simultaneously. The odd and repeating object positions were counterbalanced across the control and configural conditions. All rats received eight counterbalanced trials, four configural and four control trials.

\section{General procedure}

All rats were habituated in two consecutive daily sessions in which they were allowed to explore the empty oddity apparatus for $5 \mathrm{~min}$. For these habituation sessions, the rat was placed in the triangular apparatus, facing the corner of the triangle away from the stimuli. The experimenter did not begin timing the trial until the rat turned around from the corner of the apparatus and entered into the main exploration area. Testing began $24 \mathrm{~h}$ after the second habituation session. Rats were given a series of test trials (one per day) with a minimum interval of $24 \mathrm{~h}$ between trials. The time spent exploring objects was assessed from video recordings of the testing trial. Data were collected by scoring exploratory bouts using a personal computer running a program written in Visual Basic 6.0 (Microsoft).

\section{Configural oddity test}

All object sets used in a given trial were placed in the apparatus before the rat was placed in the apparatus. The rat was placed in the corner of the triangular apparatus (away from the stimuli), and the trial did not begin until the rat turned around and entered the main exploration area. The time spent exploring the five objects during a testing phase was scored by an experimenter viewing the rat on a video screen. The cumulative duration of exploratory bouts, the beginning and end of which were indicated by pressing a given key on the computer keyboard, was calculated by the computer program. Exploration of an object was defined as directing the nose to the object at a distance of $<2$ $\mathrm{cm}$ and/or touching it with the nose.

Exploration of the five objects in the array was recorded for $5 \mathrm{~min}$. We calculated an oddity preference score, the exploration of the odd object divided by the total exploration of the odd and identical objects. Using this score, an odd object preference of $20.0 \%$ would indicate chance-level performance (the rat explored all objects equally). An oddity preference score of $100.0 \%$ would indicate that the rat explored the odd object only. Such a score could not, however, be taken as maximum, as the rat must explore all objects before preference for the odd object occurs. Therefore, an oddity preference score significantly above $20.0 \%$ (or chance performance) would represent a meaningful score on this task.

\section{Data analysis}

Total object exploration during the oddity task (total exploration of the odd and the two identical object pairs) and a preference for the odd object (exploration of the odd object divided by total exploration) were analyzed. Means from each of these measures were submitted to a two-way ANOVA where the first factor was the between-subjects factor of lesion group and the second factor was the within-subjects factor of condition. Significant interaction effects were further analyzed with independent samples ttests. All tests of significance were performed at $\alpha=0.05$.

\section{Acknowledgments}

This work was supported by a Wellcome Trust Project Grant to T.J.B. and L.M.S. and a BBSRC Grant to T.J.B, L.M.S, and B.D.W. R.A.C. was additionally supported by European Commission Sixth Framework NEST Grant no. 516542. S.J.B. was additionally supported by a Ruth L. Kirschstein Predoctoral Fellowship from the National Institutes of Mental Health. We thank Madeline
Eacott for comments on sections of an earlier version of this manuscript.

\section{References}

Aggleton, J.P., Keen, S., Warburton, E.C., and Bussey, T.J. 1997. Extensive cytotoxic lesions involving both the rhinal cortices and area TE impair recognition but spare spatial alternation in the rat. Brain Res. Bull. 43: 279-287.

Alvarado, M.C. and Rudy, J.W. 1992. Some properties of configural learning: An investigation of the transverse-patterning problem. J. Exp. Psychol. Anim. Behav. Process. 18: 145-153.

Alvarado, M.C. and Rudy, J.W. 1995. A comparison of kainic acid plus colchicine and ibotenic acid-induced hippocampal formation damage on four configural tasks in rats. Behav. Neurosci. 109: 1052-1062.

Bartko, S.J., Winters, B.D., Cowell, R.A., Saksida, L.M., and Bussey, T.J. 2007. Perceptual functions of perirhinal cortex in rats: Zero-delay object recognition and simultaneous oddity discriminations. J. Neurosci. 27: 2548-2559.

Bogacz, R., Brown, M.W., and Giraud-Carrier, C. 2001. Model of familiarity discrimination in the perirhinal cortex. J. Comput. Neurosci. 10: 5-23.

Buckley, M.J. and Gaffan, D. 1998. Perirhinal cortex ablation impairs configural learning and paired- associate learning equally. Neuropsychologia 36: 535-546.

Buffalo, E.A., Ramus, S.J., Clark, R.E., Teng, E., Squire, L.R., and Zola, S.M. 1999. Dissociation between the effects of damage to perirhinal cortex and area TE. Learn. Mem. 6: 572-599.

Buffalo, E.A., Ramus, S.J., Squire, L.R., and Zola, S.M. 2000. Perception and recognition memory in monkeys following lesions of area TE and perirhinal cortex. Learn. Mem. 7: 375-382.

Burwell, R.D. 2001. Borders and cytoarchitecture of the perirhinal and postrhinal cortices in the rat. J. Comp. Neurol. 437: 17-41.

Bussey, T.J. and Saksida, L.M. 2002. The organization of visual object representations: A connectionist model of effects of lesions in perirhinal cortex. Eur. J. Neurosci. 15: 355-364.

Bussey, T.J. and Saksida, L.M. 2005. Object memory and perception in the medial temporal lobe: An alternative approach. Curr. Opin. Neurobiol. 15: 730-737.

Bussey, T.J. and Saksida, L.M. 2007. Memory, perception, and the ventral visual-perirhinal-hippocampal stream: Thinking outside of the boxes. Hippocampus 17: 898-908.

Bussey, T.J., Muir, J.L., and Aggleton, J.P. 1999. Functionally dissociating aspects of event memory: The effects of combined perirhinal and postrhinal cortex lesions on object and place memory in the rat. $J$. Neurosci. 19: 495-502.

Bussey, T.J., Duck, J., Muir, J.L., and Aggleton, J.P. 2000. Distinct patterns of behavioural impairments resulting from fornix transection or neurotoxic lesions of the perirhinal and postrhinal cortices in the rat. Behav. Brain Res. 111: 187-202.

Bussey, T.J., Saksida, L.M., and Murray, E.A. 2002. Perirhinal cortex resolves feature ambiguity in complex visual discriminations. Eur. J. Neurosci. 15: 365-374.

Bussey, T.J., Saksida, L.M., and Murray, E.A. 2003. Impairments in visual discrimination after perirhinal cortex lesions: Testing 'declarative' vs. 'perceptual-mnemonic' views of perirhinal cortex function. Eur. J. Neurosci. 17: 649-660.

Cowell, R.A., Bussey, T.J., and Saksida, L.M. 2006. Why does brain damage impair memory? A connectionist model of object recognition memory in perirhinal cortex. J. Neurosci. 26: 12186-12197.

Dix, S.L. and Aggleton, J.P. 1999. Extending the spontaneous preference test of recognition: Evidence of object-location and object-context recognition. Behav. Brain Res. 99: 191-200.

Eacott, M.J., Gaffan, D., and Murray, E.A. 1994. Preserved recognition memory for small sets, and impaired stimulus identification for large sets, following rhinal cortex ablations in monkeys. Eur. J. Neurosci. 6: $1466-1478$.

Eacott, M.J., Machin, P.E., and Gaffan, E.A. 2001. Elemental and configural visual discrimination learning following lesions to perirhinal cortex in the rat. Behav. Brain Res. 124: 55-70.

Forwood, S.E., Winters, B.D., and Bussey, T.J. 2005. Hippocampal lesions that abolish spatial maze performance spare object recognition memory at delays of up to 48 hours. Hippocampus 15: 347-355.

Glenn, M.J. and Mumby, D.G. 1998. Place memory is intact in rats with perirhinal cortex lesions. Behav. Neurosci. 112: 1353-1365.

Holdstock, J.S., Gutnikov, S.A., Gaffan, D., and Mayes, A.R. 2000. Perceptual and mnemonic matching-to-sample in humans: Contributions of the hippocampus, perirhinal and other medial temporal lobe cortices. Cortex 36: 301-322.

Jenkins, T.A., Amin, E., Pearce, J.M., Brown, M.W., and Aggleton, J.P. 
2004. Novel spatial arrangements of familiar visual stimuli promote activity in the rat hippocampal formation but not the parahippocampal cortices: A c-fos expression study. Neuroscience 124: $43-52$.

Levy, D.A., Shrager, Y., and Squire, L.R. 2005. Intact visual discrimination of complex and feature-ambiguous stimuli in the absence of perirhinal cortex. Learn. Mem. 12: 61-66.

Meunier, M., Bachevalier, J., Mishkin, M., and Murray, E.A. 1993. Effects on visual recognition of combined and separate ablations of the entorhinal and perirhinal cortex in rhesus monkeys. J. Neurosci. 13: $5418-5432$.

Mumby, D.G. and Pinel, J.P. 1994. Rhinal cortex lesions and object recognition in rats. Behav. Neurosci. 108: 11-18.

Murray, E.A., Bussey, T.J., and Saksida, L.M. 2007. Visual perception and memory: A new view of medial temporal lobe function in primates and rodents. Annu. Rev. Neurosci. 30: 99-122.

Norman, G. and Eacott, M.J. 2004. Impaired object recognition with increasing levels of feature ambiguity in rats with perirhinal cortex lesions. Behav. Brain Res. 148: 79-91.

Norman, G. and Eacott, M.J. 2005. Dissociable effects of lesions to the perirhinal cortex and the postrhinal cortex on memory for context and objects in rats. Behav. Neurosci. 119: 557-566.

Norman, K.A. and O'Reilly, R.C. 2003. Modeling hippocampal and neocortical contributions to recognition memory: A complementary-learning-systems approach. Psychol. Rev. 110: $611-646$.

Paxinos, G. and Watson, C. 1998. The rat brain in stereotaxic coordinates. Academic Press, London, UK.

Pearce, J.M. 1994. Similarity and discrimination: A selective review and a connectionist model. Psychol. Rev. 101: 587-607.
Rudy, J.W. and Sutherland, R.J. 1989. The hippocampal formation is necessary for rats to learn and remember configural discriminations. Behav. Brain Res. 34: 97-109.

Saksida, L.M., Bussey, T.J., Buckmaster, C.A., and Murray, E.A. 2007. Impairment and facilitation of transverse patterning after lesions of the perirhinal cortex and hippocampus, respectively. Cereb. Cortex 17: 108-115.

Sutherland, R.J. and Rudy, J.W. 1989. Configural association theory: The role of the hippocampal formation in learning, memory, and amnesia. Psychobiology 17: 129-144.

Suzuki, W.A., Zola-Morgan, S., Squire, L.R., and Amaral, D.G. 1993. Lesions of the perirhinal and parahippocampal cortices in the monkey produce long-lasting memory impairment in the visual and tactual modalities. J. Neurosci. 13: 2430-2451.

Wan, H., Aggleton, J.P., and Brown, M.W. 1999. Different contributions of the hippocampus and perirhinal cortex to recognition memory. J. Neurosci. 19: 1142-1148.

Winters, B.D. and Bussey, T.J. 2005. Glutamate receptors in perirhinal cortex mediate encoding, retrieval, and consolidation of object recognition memory. J. Neurosci. 25: 4243-4251.

Winters, B.D., Forwood, S.E., Cowell, R.A., Saksida, L.M., and Bussey, T.J. 2004. Double dissociation between the effects of peri-postrhinal cortex and hippocampal lesions on tests of object recognition and spatial memory: Heterogeneity of function within the temporal lobe. J. Neurosci. 24: 5901-5908.

Received August 21, 2007; accepted in revised form October 20, 2007. 


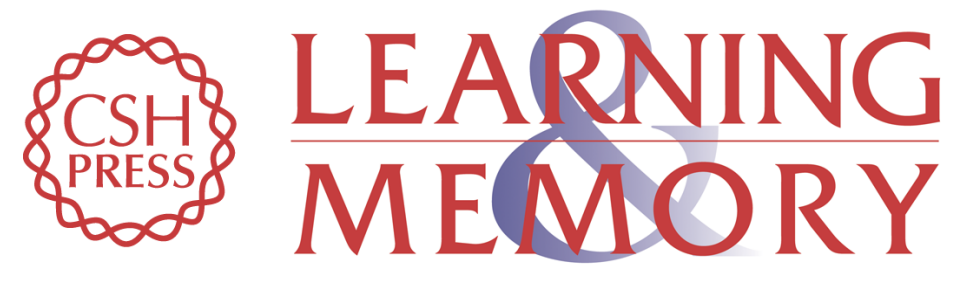

\title{
Perirhinal cortex resolves feature ambiguity in configural object recognition and perceptual oddity tasks
}

\author{
Susan J. Bartko, Boyer D. Winters, Rosemary A. Cowell, et al.
}

Learn. Mem. 2007, 14:

Access the most recent version at doi:10.1101//m.749207

References

License

\section{Email Alerting} Service
This article cites 39 articles, 11 of which can be accessed free at: http://learnmem.cshlp.org/content/14/12/821.full.html\#ref-list-1

Receive free email alerts when new articles cite this article - sign up in the box at the top right corner of the article or click here. 